\title{
Analysis of Manipulator Structures under Joint-Failure with Respect to Efficient Control in Task-Specific Contexts
}

\author{
Susanne Petsch and Darius Burschka
}

\begin{abstract}
Robots are meanwhile able to perform several tasks. But what happens, if one or multiple of the robot's joints fail? Is the robot still able to perform the required tasks? Which capabilities of the robot get limited and which ones are lost?

We propose an analysis of manipulator structures for the comparison of a robot's capabilities with respect to efficient control. The comparison is processed (1) within a robot in the case of joint failures and (2) between robots with or without joint failures. It is important, that the analysis can be processed independently of the structure of the manipulator. The results have to be comparable between different manipulator structures. Therefore, an abstract representation of the robot's dynamic capabilities is necessary. We introduce the Maneuverability Volume and the Spinning Pencil for this purpose. The Maneuverability Volume shows, how efficiently the endeffector can be moved to any other position. The Spinning Pencil reflects the robot's capability to change its end-effector orientation efficiently.

Our experiments show not only the different capabilities of two manipulator structures, but also the change of the capabilities if one or multiple joints fail.
\end{abstract}

\section{Motivation}

Today, robots are able to perform different tasks as, e.g., work in the kitchen. The structure of the used robots can be very different, reaching from, e.g., a simple three Degrees-of-Freedom (DoF) manipulator to complex systems like humanoid robots. Hence, the robot's capabilities can vary strongly. Some of the robots might have extensive capabilities, whereas others have just a limited ones. The efficiency during the desired motion can be different as well. In an ideal case, the robot moves smoothly and slowly. The workload should be distributed equally among all joints.

Our aim is the comparison of different manipulator structures with respect to efficient control. We want to use one approach which leads to a result that is independent of the structure of the robot. At the same time, the change of the manipulator's capabilities should be analyzable if one or several of its joints fail. Is the robot able to reach the desired positions at all? Which capabilities of the manipulator remain at which quality and which ones are lost?

Hence, we are interested in an abstraction of the robot's capabilities, which enables an inter-robot and intra-robot comparison of the efficiency in dynamics. The possibly high dimensionality of the manipulator structure has to be reduced to a representation at a manageable dimensionality. Fig. 1

This work was supported by a cooperation with the German Aerospace Center (DLR)

Susanne Petsch and Darius Burschka are with the Machine Vision and Perception Group, Department of Informatics, Technische Universität München. 85748 Garching, Germany

\{petsch, burschka\}ein.tum.de

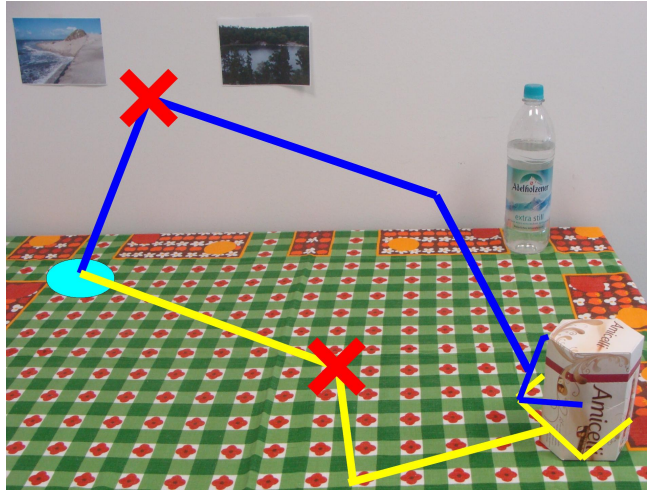

Fig. 1. Analysis of manipulator structures under joint failure (light blue circle: robot's base): What happens, if, e.g., the red-marked second joint of the blue manipulator fails? Is the robot still able to move the box as a task might require? If yes, which effort is necessary? Can the robot still turn the object? How would the yellow robot be affected under a similar joint failure? Would it keep better capabilities than the first one?

illustrates an exemplary scene with two manipulators, which are supposed to perform the same task under joint failure.

We introduce the Maneuverability Volume and the Spinning Pencil as abstract representation of the robot's capabilities for the inter-robot and intra-robot comparison.

The Maneuverability Volume is a parallelepiped, which represents the robot's capabilities to move efficiently into a set of directions. The starting point of our idea is the following: If a joint is moved by a fixed angular rate, it contributes a certain velocity to the end-effector, which is represented by a base velocity vector. The base velocity vectors of all joints can be combined to the desired overall velocity of the end-effector. Of course, it is desirable, that the base velocity vectors point into significantly different directions to enable an efficient motion into a large set of directions. Hence, the vectors should span a large volume to cover a large area. Moreover, the vectors are spanned by the fixed angular rate, which is the same for all joints. Therefore, the magnitudes of the base velocity vectors allow an easy comparison of the efficiency of different joints. A joint which contributes a vector of a large magnitude is more efficient than another joint contributing a vector of a smaller magnitude.

The robot's capability to change its end-effector position efficiently is represented by the described Maneuverability Volume. We want to be able to separately analyze the robot's capabilities to change the end-effector orientation efficiently. In an ideal case, the robot is able to turn its endeffector without changing its position. Otherwise, a change of orientation requires also a correction of the position. For 
example, the robot could have to rotate an object without changing the position (e.g., a jug needs to be turned to fill coffee into a cup). We can imagine the axis of the desired rotation as the rotation-axis of a spinning top. If an undesired change in position comes along with the change in orientation, we need to model it. We take the change in position of the end-effector as the spinning top's diameter. A spinning top in form of a spinning pencil is desirable, since the pencil reflects the advantageous thin shape of the spinning top. Hence, we represent the robot's capability to change its end-effector orientation by the Spinning Pencil.

We are interested in the analysis of the manipulator structure in a task-specific context. The representation of the task is important to us, since it is the basis on which we work. We want to use an efficient, abstract representation of a task. Therefore, we develop the concept of Functionality Maps and Location Areas in [1] further. We make use of the Location Areas as representations of positions, where actions typically start or end. In some areas, only simple tasks are performed. These tasks can even be performed by a robot with limited capabilities. In contrast, complex manipulations can take place at other areas. Consequently, the task determines the areas where manipulations are performed and the capabilities which are required from the robot. For example, a Location Area in a cupboard is used to place an object mainly from one direction, which is the front. In contrast, complex manipulations can be performed at a Location Area on a table. The robot needs better capabilities on the table than at the cupboard. The capabilities which are required by the desired, abstract task need to be integrated into the analysis.

The paper is structured as follows: After an overview of the related work, the approach is presented. In the approach, the Maneuverability Volume and the Spinning Pencil are described first. The Maneuverability Analysis under joint failures follows afterward. The extension of the approach to multiple Location Areas is the last part of the approach. The implementation, the data and the results of our experiments are described thereafter. We end with conclusions.

\section{RELATED WORK}

The Jacobian Matrix is often incorporated into the analysis of manipulators. For example, the manipulability measure in [2] is based on the determinant of the Jacobian Matrices. Kim and Khosla [3] investigate further in the Jacobian as dexterity measure of a manipulator. They introduce the measure of isotropy, which can be used to develop a manipulator which is close to an isotropic configuration for a given position in 3D space. Reachability and joint limits are considered for the kinematic design of serial link manipulators in [4].

Asada [5] proposes a dynamic analysis of a manipulator's workspace based on a graphical representation of inertia ellipsoids. Lee [6] compares manipulability ellipsoids and manipulability polytopes. The Dynamic Capability Equations in [7] describe the acceleration and force capabilities of a robot at a particular configuration under the consideration of the limitations of the manipulator's motor torques. The translational and rotational quantities are mapped into representations of actuator torques with same units.

Dimensionality reduction techniques are presented for different robotic applications, e.g., grasping. In [8], a dimensionality reduction is used for hand-independent dexterous robotics grasping. The spatial and temporal context of human grasping actions is studied in [9].

In contrast to the described approaches, we aim to analyze any arbitrary manipulator with respect to the change of the efficiency of its dynamics under possible joint failures in the context of an abstractly represented task. As already described in the Motivation, we are interested in the interand intra-robot analysis of the efficiency of the robot's motion, the variety of directions in which the end-effector can be moved efficiently and the capability to change the orientation of the end-effector without a change in position. The manipulator can have an arbitrary number of joints. Therefore, it could be highly redundant, too. It is important to notice, that we do not aspire to model torque bounds or something similar. The analysis of parallel manipulators (e.g., [10]) is not our aim, we focus on serial link manipulators.

\section{APPROACH}

We describe the robotic system in the DH-convention suggested by Denavit and Hartenberg [11] in the form shown in [12]. The orientation of the robot's end-effector is described as Z-Y-X Euler angles [12]. The overall number of joints is $N$.

\section{A. Maneuverability Volume}

At a (reachable) position, the robot can move its endeffector into a larger or a smaller set of directions depending on the design and the current configuration. The content of the set depends on the motion of each joint, since each joint $i$ contributes a certain linear velocity vector $v_{i}$ to the overall velocity $v=\sum_{i} v_{i}$ of the end-effector. All vectors in this paper are given in a global coordinate frame, if not labeled otherwise. The linear velocity $v_{i}$ of a rotational joint $i$ can be computed by

$$
v_{i}=\omega_{i} \times\left(P_{e e}-P_{i}\right)
$$

with $P_{e e}$ as the position of the end-effector, $P_{i}$ as the position of joint $i$ and $\omega_{i}$ as the rotational velocity caused by joint $i$ :

$$
\omega_{i}=\dot{\theta}_{i} \cdot \hat{Z}_{i}
$$

with $\hat{Z}_{i}$ as 3-dimensional unit vector of the z-axis of joint $i$ and $\dot{\theta}_{i}$ as magnitude of the angular rate (see, e.g., [12]).

If we consider a fixed angular rate $\dot{\theta}_{f i x}$ for each joint $i$, the magnitude of the rotational velocity $\omega_{i, f i x}$ is the same for all joints, since $\hat{Z}_{i}$ is a unit vector. The resulting linear velocity is called base velocity $d_{i}$ of a joint $i$. It depends on the current configuration:

$$
d_{i}=\left(\dot{\theta}_{f i x} \cdot \hat{Z}_{i}\right) \times\left(P_{e e}-P_{i}\right)
$$

or

$$
d_{i}=\omega_{i, f i x} \times\left(P_{e e}-P_{i}\right)
$$




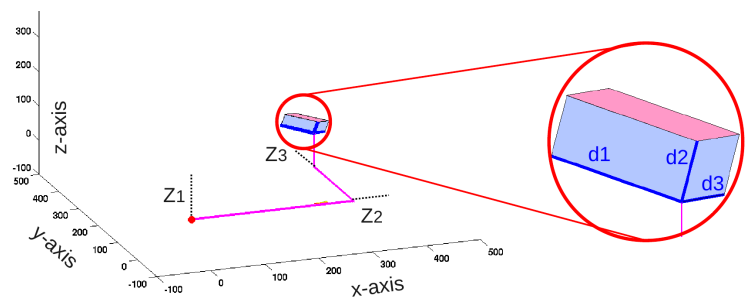

Fig. 2. Parallelepiped (part $K$ of the Maneuverability Volume): A robot with three rotational joints is drawn in magenta. Its joints can turn around the black dotted $z_{i}$-axes. Each joint contributes one of the blue base velocity vectors $d_{i}$, when it moves with the speed $\dot{\theta}_{f i x}$. E.g., the largest vector $d_{1}$ is contributed by joint 1 . The three blue base velocity vectors $d_{i}$ span the light blue/ light red parallelepiped. The red dot is the robot's base.

A desired end-effector speed can be combined by the base velocities:

$$
v=\sum_{i} v_{i}=\sum_{i}\left(g_{i} \cdot d_{i}\right)
$$

with a scalar $g_{i}$. Of course, it depends strongly on the vectors of the base velocity, whether and at which effort a desired end-effector speed can be achieved. If low joint speeds are desired, the base velocity vectors should have a large magnitude (resulting in a small effort $g_{i}$ in Eq. 5). Moreover, the vectors $d_{i}$ (and their counterparts, pointing in the opposite direction) should be perpendicular to each other. A combination of the vectors $d_{i}$ can, then, efficiently reach any direction in space. At the same time, the robot is further away from a singular configuration. Such a singularity would be reached, if at least two vectors point into the same direction.

Three base velocity vectors span a volume (parallelepiped), which increases, when the vectors are rather perpendicular to each other and when the magnitude of the vectors is enlarged. A parallelepiped can be computed by a triple product $V=\operatorname{trip}\left(d_{1}, d_{2}, d_{3}\right)=\left(d_{1} \times d_{2}\right) \cdot d_{2}$. The parallelepiped $K$ of three base velocity vectors is therefore:

$$
K=\operatorname{trip}\left(d_{1}, d_{2}, d_{3}\right)
$$

An advantageous parallelepiped has a large value $K$.

Moreover, an evaluation of the maneuverability independently of the length of the base velocity vectors $d_{i}$ is interesting, since we can ensure that the base velocity vectors are perpendicular to each other as good as possible. First, a normalization is executed: $\hat{d}_{l}=\frac{d_{l}}{\left\|d_{l}\right\|}$. Afterward, just the angles between the vectors are considered in the parallelepiped $L$ :

$$
L=\operatorname{trip}\left(\hat{d}_{1}, \hat{d}_{2}, \hat{d}_{3}\right)
$$

The combination of both parallelepiped $K$ and $L$ gives us the desired measurement of maneuverability, which we call the Maneuverability Volume $m V$ :

$$
m V=\omega_{k} \cdot \operatorname{trip}\left(d_{1}, d_{2}, d_{3}\right)+\omega_{l} \cdot \operatorname{trip}\left(\hat{d}_{1}, \hat{d}_{2}, \hat{d}_{3}\right)
$$

with the weightings $\omega_{k}$ and $\omega_{l}$ to enable different priorities of the desired properties. An examplary parallelepiped $K$ is illustrated in Fig. 2.

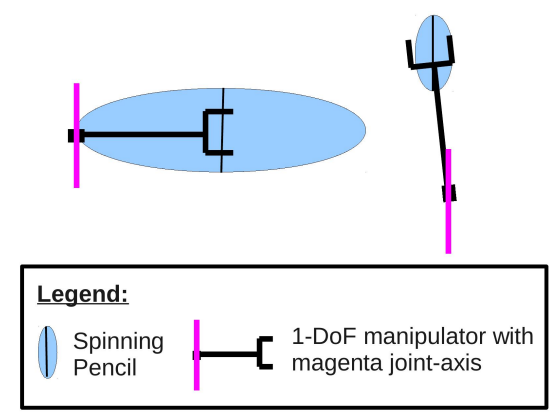

Fig. 3. Spinning Pencil. Two different 1-DoF manipulators are illustrated. The thin black line in the blue Spinning Pencil shows the length and the orientation of the pencil. Both pencils have the same length and point into the same direction. The different magnitudes of the diameters are due to the fact, that the end-effector on the left conquers a much larger distance to change its orientation than the end-effector on the right.

The Maneuverability Volume can be spanned by three vectors. If the robot has more than three joints, we combine the base velocity vectors $d_{i}$ to three final velocity vectors $d_{i, f}$. We just need to check all combinations of the vectors $d_{i}$ to the final $d_{i, f}$ to find the most advantageous Maneuverability Volume. For example, six base velocity vectors $d_{i}$ should be combined to three final velocity vectors. One final velocity vector $d_{1, f}$ can consist of one, two, three or four different vectors $d_{i}$, so that the remaining two final velocity vectors $d_{i, f}$ consist of at least one vector $d_{i}$. Each vector $d_{i}$ can just be assigned to one of the final velocity vectors $d_{i, f}$. Consequently, our analysis takes place in the 3D-space, where the manipulations takes actually place. A projection to another space is not necessary.

In contrast to the traditional approach with the determinant of a Jacobian, we are include in our Maneuverability Volume (1) an additional emphasis on the desired $90^{\circ}$ angle between the base velocity vectors and (2) a new extension to manipulators with more than three DoF.

\section{B. Spinning Pencil}

The next step is the analysis of a manipulator's capability to change its end-effector orientation efficiently. It is desirable, that the orientation can be changed around any axis at the position of the end-effector, while the position of the end-effector is not changing. For example, a jug needs to be turned around a certain axis to fill a cup with coffee, while the center of the jug should stay at its original position. As already described in the Motivation, we imagine a spinning top, which reflects these properties: The spinning top rotates around a certain axis. In the case of a manipulator, the endeffector can turn around one (or more) of its joint axis. Depending on the robot's configuration, a change of the orientation can automatically and unpreventably result in an undesired position change. The distance between the position of the end-effector before and after the rotation is chosen as the diameter of the spinning top. A small diameter is, of course, desirable. Hence, we introduce the Spinning Pencil, since the pencil reflects the small diameter. Fig. 3 illustrates the principle of the Spinning Pencil. 
We want to analyze the robot's capabilities to change the orientation of its end-effector efficiently, while keeping the desired position $P_{e e}$. Therefore, we turn joint $i$ by an angle $\gamma$. In an ideal case, the end-effector stays at $P_{e e}$ independently of the magnitude of $\gamma$. Otherwise, the end-effector moves to a new position $P_{n, i}$. The euclidean distance between the old position $P_{e e}$ and the new one $P_{n, i}$ is labeled with $e_{i}=$ $P_{n, i}-P_{e e}$. We compute the mean $\mu_{e}$ and the variance $\sigma_{e}$ of all $e_{i}$ (all joints $i$ ), which should be small in an advantageous Spinning Pencil.

Of course, the robot should be able to change the endeffector's orientation around any arbitrary axis running through the original position $P_{e e}$. However, the robot's capability to turn depends on the z-axes of its joints. Hence, $\mathrm{z}$-axes which point nearly in the same direction provide almost the same axis for the change of orientation. The more different the orientation of the z-axes, the more axes are provided for the orientation change. In the ideal case, the $\mathrm{z}-$ axes are perpendicular to each other. Therefore, we compute the normalized z-axis ${ }^{e e} \hat{Z}_{i}$ of each joint $i$ in the frame of the end-effector. Afterward, we analyze the angles between the z-axes ${ }^{e e} \hat{Z}_{i}$.

Once again, we want to process the analysis in 3D space, where the manipulations actually take place. Since the manipulator can be redundant, it might be necessary to analyze more than three z-axes. Hence, we analyze the possible combinations of z-axes. We compute the determinant of three normalized axes, since it reflects the angles between the axes: If all three axes are perpendicular to each other, the determinant reaches its maximum of one. The smaller the determinant, the less perpendicular the axes are to each other. Therefore, we take a combination $c$ of three z-axes ${ }^{e e} \hat{Z}_{i}$ and compute the determinant $s_{1, c}$ :

$$
s_{1, c}=\operatorname{det}\left({ }^{e e} \hat{Z}_{c 1},{ }^{e e} \hat{Z}_{c 2},{ }^{e e} \hat{Z}_{c 3}\right)
$$

with $c 1$, etc., as labeling of the three chosen z-axes ${ }^{e e} \hat{Z}_{i}$. The determinant $s_{1, c}$ is computed for all $n_{1}=\left(\begin{array}{c}N \\ 3\end{array}\right)$ combinations. All determinants $s_{1, c}$ are summed up and normalized with the overall number of combinations $n_{1}$ :

$$
s_{1}=\frac{\sum_{c} s_{1, c}}{n_{1}}
$$

It is possible, that at least some $\mathrm{z}$-axes lie in a plane. In this case, the determinant in Eq. 9 becomes zero. Therefore, we analyze the angle between two z-axes additionally. The dot product is used for this purpose. Similarly to $s_{1, c}$, we compute the dot product $s_{2, c}$ for all combinations of two z-axes:

$$
s_{2, c}={ }^{e e} \hat{Z}_{c 1} \cdot{ }^{e e} \hat{Z}_{c 2}
$$

The results of $s_{2, c}$ are projected into the range of $0-90^{\circ}$. The final $s_{2}$ consists of the sum of all $s_{2, c}$, which is normalized with the total number of combinations $n_{2}=\left(\begin{array}{c}N \\ 2\end{array}\right)$ :

$$
s_{2}=\frac{\sum_{c} s_{2, c}}{n_{2}}
$$

The values of $s_{1}$ and $s_{2}$ should be large in an advantageous case. In contrast, the mean $\mu_{e}$ and the variance $\sigma_{e}$ should be minimized. Therefore, we combine all parts of the Spinning Pencil $s P$ as follows:

$$
s P=\omega_{1} \cdot s_{1}+\omega_{2} \cdot s_{2}+\omega_{3} \cdot \frac{1.0}{\mu_{e}+\sigma_{e}}
$$

with $\omega_{i}$ as weighting of the respective property. All terms of the $s P$ should be large in an advantageous case. The third term $\frac{1.0}{\mu_{e}+\sigma_{e}}$ is increasing, when the mean $\mu_{e}$ and the variance $\sigma_{e}$ are decreasing as desired.

In contrast to traditional approaches with the Jacobian, we consider also (1) rotation axes lying in a plane and (2) the undesired effect of a position change which can come along with a change in orientation.

\section{Maneuverability Analysis under Joint-Failures}

Next, we want to analyze the remaining maneuverability of a manipulator, if one or more of its joints fail. First, we compute the original maneuverability. We combine the Maneuverability Volume and the Spinning Pencil in the Maneuverability Analysis $M A_{l}$ at Location Area l:

$$
M A_{l}=\omega_{m} \cdot m V+\omega_{s} \cdot s P
$$

with a weighting $\omega_{m}$ and $\omega_{s}$ to enable different preferences of both properties (sum of $\omega_{m}$ and $\omega_{s}$ equals 1.0).

After the computation of the original $M A_{l}$, the Maneuverability Analysis is processed for the manipulator with the broken joint. The manipulator with the broken joint has simply one DoF less than the original one. The DH-parameters need just to be changed around the broken joint: A direct transformation from the joint before the broken joint to the joint after the broken joint is needed. The joint angle $\theta_{b}$ of the broken joint $b$ can be treated as a fixed parameter. We just need to process the above Maneuverability Analysis for the new manipulator, since it can be computed for manipulators with any number of joints. Therefore, the procedure can also be easily extended to a manipulator with multiple broken joints.

\section{Application on Multiple Location Areas}

Up to now, we described the approach for one Location Area. Most of the times, the robot has to be able to work at multiple Location Areas. The requirements on the maneuverability depend on the type of Location Area. Therefore, we introduce a weighting $\omega_{l}$ of each Location Area $l$ depending on the level of the required maneuverability. A high maneuverability requirement corresponds to a high weighting of the Location Area.

The final objective function $M A$ contains the Maneuverability Analysis $M A_{l}$ of each Location Areas $l$ :

$$
M A=\sum_{l} \omega_{l} \cdot M A_{l}
$$

with the weighting $\omega_{l}$ of Location Area $l$. The higher the final value $M A$, the better the maneuverability

\section{EXPERIMENTS}

The context of our experiments is a manipulation task. We analyze the robot's capabilities under the failure of none, one or multiple joints. 

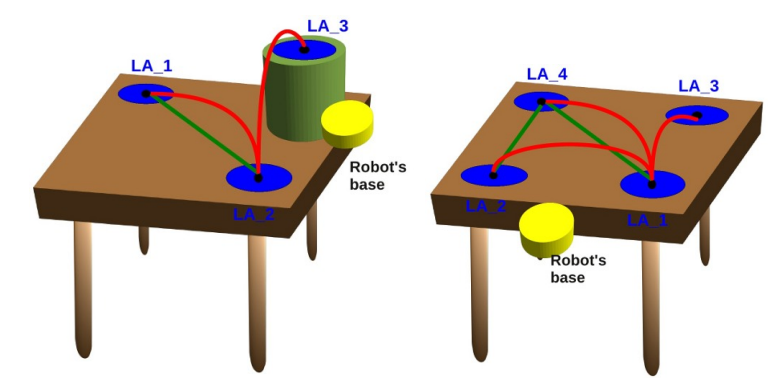

Fig. 4. Data set I (left) and II (right): The lines/ curves between the blue Location Areas refer to trajectories of lifted objects (red) and trajectories of pushed objects (green). The robot's base positions are symbolized in yellow.

\section{A. Data and Implementation}

The implementation is done in $\mathrm{C} / \mathrm{C}++$. The estimation of the inverse kinematics is done through a stochastic approach for global minimization presented in [13]. We use the implementation by Oliver Ruepp [14]. The data consists of two data sets, which were extracted from observations of humans in [1]. Both data sets are illustrated in Fig. 4.

We process the Maneuverability Analysis for two different 6-DoF manipulators. The first one is an arbitrary robot, which has simply consecutive rotational joints perpendicular to each other and all links have a length of $300 \mathrm{~mm}$ (DHParameter: $\left.d_{i}, a_{5}\right)$. The other manipulator is a widely known Unimation PUMA 560. The robot's base positions are shown in Fig. 4. The orientation of the base is chosen in a manner, such that the joint axis of the first joint is perpendicular to the expected main plane of manipulation (e.g., table plane).

We use a fixed angular rate of $\dot{\theta}_{f i x}=1.0 \mathrm{rad}$ to compute the Maneuverability Volume. Moreover, we focus on the parallelepiped $K$ of the Maneuverability Volume (Eq. 8). Therefore, it is prioritized with a weighting $\omega_{k}=0.8$ in contrast to $\omega_{l}=0.2$. For the Spinning Pencil, the angle $\gamma$ is set to $180^{\circ}$, considering the maximally crossed distance in the turn of a joint in the worst case. The weightings in Eq. 13 are chosen as $\omega_{1}=\frac{1}{12}, \omega_{2}=\frac{1}{12}$ and $\omega_{3}=\frac{5}{6}$, which leads to an equal weighting of the first two terms and an emphasis on the third term in the equation. The third term is important to us, since it supports the desired thin Spinning Pencil. We set a strong priority on the Maneuverability Volume in the Maneuverability Analysis $M A_{l}$ with $\omega_{m}=0.75$ and $\omega_{s}=0.25$, since we prioritize the capability to move the object in the manipulation experiment.

Concerning the weighting of the Location Areas, we choose the following priorities. Characteristic area 2 in data set $\mathrm{I}$ is chosen as the most important area with a weighting of 0.5. It is located on the table and can, therefore, be used for more, and also more complicated manipulations than the other Location Areas. These act as places of storage and have a weighting factor of 0.25 . The weighting of the Location Areas in data set II is related to the number of inand out-coming trajectories. The more in- and out-coming trajectories, the higher the weighting.

\section{B. Results}

We perform experiments with no, one, two and three broken joints. The end-effector is able to reach a desired position, if the remaining distance between the desired and the real position is smaller than a tolerance $t$. The remaining distance is a city block distance $p=p_{p}+\alpha \cdot p_{o}$ with the distance $p_{p}$ in 3D position (unit: $\mathrm{mm}$ ) and the distance $p_{o}$ in orientation (unit: $\mathrm{rad}$ ). The variable $\alpha$ is used to compensate the different scales of $p_{p}$ and $p_{o}$ ( $\alpha=300$ in our experiments). The tolerance $t$ is set to 25 in the experiments with no, resp., one broken joint. A higher tolerance $t=150$ is necessary in the experiments with two or three broken joints, in order to be able to reach the desired positions at all before the remaining maneuverability can be analyzed. This shows already, that the failure of multiple joints influences the capabilities of the robots significantly, since the robot is often not even able to reach a desired position. If several configurations are possible at the desired position, we analyze all of them and take the most advantageous one according to the Maneuverability Analysis. Hence, our experiments are not limited to the analysis of a single configuration.

At first, the Maneuverability Analysis is processed for the entirely working robots in comparison to the robots with one broken joint. As the results in Fig. 5 and 6 show, each robot has joints, which are essential to reach the desired positions. In contrast, a failure of another joint might hardly affect the performance. It is interesting, that joint 4 of the arbitrary robot is essential for both tasks. Moreover, a failure of one of the last three joints of the PUMA hardly affects the performance as in both data sets. In the following, we focus on the discussion of data set II, since both robot's have approximately the same magnitude of the results in the Maneuverability Analysis, what enables a better comparison of the joint failures. An evaluation of data set I would be done similarly.

As already mentioned, the tolerance for a hit is enlarged for the experiments with two or three broken joints. This is important for the discussion of the results for the robots with two broken joints in Fig. 7. For example, Fig. 6 shows, that the PUMA does not reach its desired position if joint 1 fails. Therefore, the PUMA should not be able to reach its desired positions if joint 1 and a further joint are broken. Due to the necessary extension of the tolerance, the PUMA is able to reach its desired position if, e.g., joint 1 and 4 are broken. Fig. 7 shows the joints, which are essential even under the extended tolerance.

Of course, we expect that a higher number of working joints increases the magnitude of the result in the Maneuverability Analysis. The comparison of the experiments with a different number of broken joints confirms this widely (see Fig. 8). One has to consider the larger tolerance for a hit in the experiments with two or three broken joints. Therefore, it is possible, that the robot can reach points in the additional tolerance with new configurations. The new configurations can be advantageous with respect to the Maneuverability Analysis. Hence, the result of the Maneuverability Analysis 


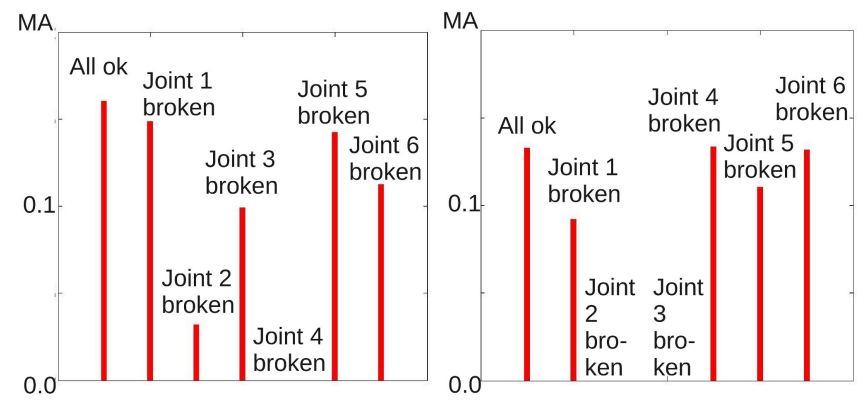

Fig. 5. Complete manipulator vs. manipulator with one broken joint for the arbitrary robot (left) and the PUMA (right), data set I. As it can be seen, there are essential joints: If these joints fail, the robot cannot reach the desired positions any more, since $M A=0$ (joint 4 of the arbitrary robot; joint 2, resp., 3 of the PUMA). The failure of others can reduce the maneuverability (e.g., joint 3 of the arbitrary robot), whereas the failure of some others hardly affects the maneuverability in our requirements (e.g., joint 4 of the PUMA). We have put an emphasis on the capability to move the end-effector rather than to turn it in our requirements. Therefore, it is quite consequential from the PUMA's structure, that the failure of one of the last three joints of the PUMA reduces the robot's maneuverability less significantly than the failure of the first three joints. The last three joints are significantly closer to the end-effector than the first three (see link lengths of the PUMA). Hence, the first three joints can be used to bridge a large gap to a desired position, whereas the last three joints can hardly perform this. It becomes clear, that especially the joint 2 and 3 are essential for the PUMA to reach the desired positions in data set I.

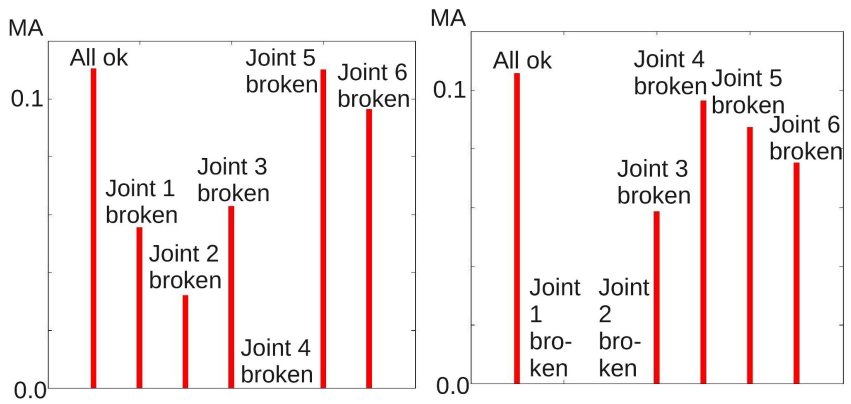

Fig. 6. Complete manipulator vs. manipulator with one broken joint for the arbitrary robot (left) and the PUMA (right), data set II. Similarly to Fig. 5, the essential joints for the task become visible.
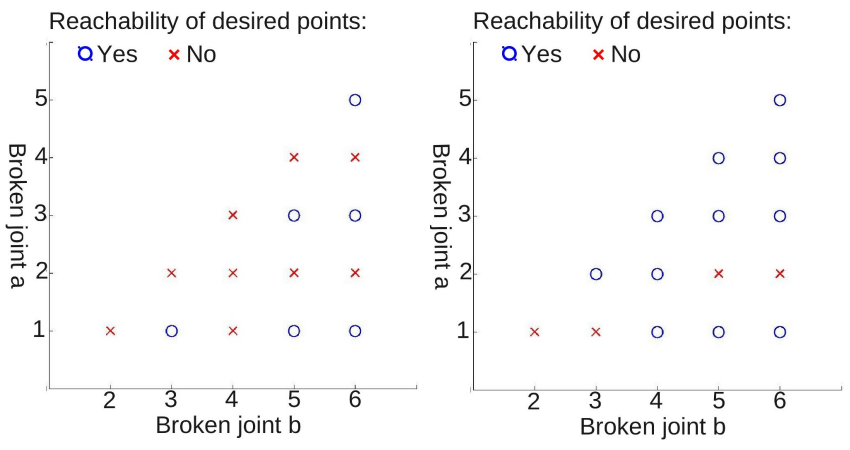

Fig. 7. Capability of the arbitrary robot (left) and the PUMA (right) to reach the desired positions with two broken joints. As it can be seen, joint 2 and 4 is very essential for the arbitrary robot to reach the positions even with the extended tolerance.

of the arbitrary robot with two broken joints can be better than with one broken joint.

Fig. 9-13 show some of the resulting Maneuverability
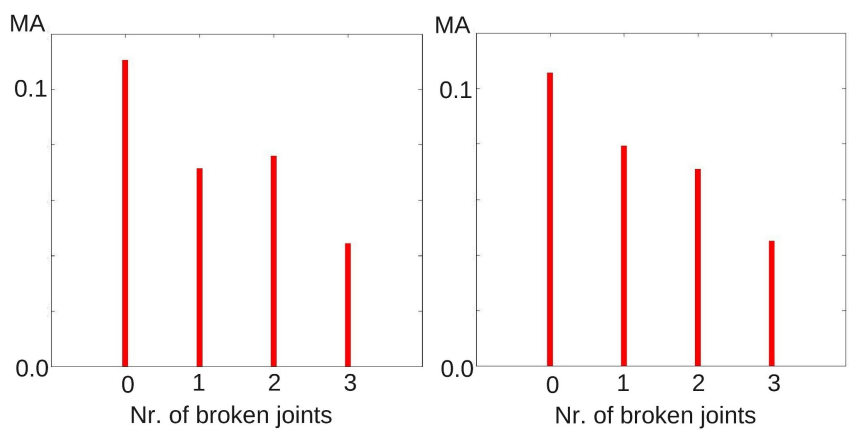

Fig. 8. Average result of the Maneuverability Analysis with respect to the number of broken joints for the arbitrary robot (left) and the PUMA (right). Most of the times, a higher number of broken joints leads to a reduced maneuverability as expected. The higher value of the Maneuverability Analysis for the arbitrary robot with two broken joints can be explained through the higher tolerance for the hit of a desired position in the experiments with two or three broken joints.
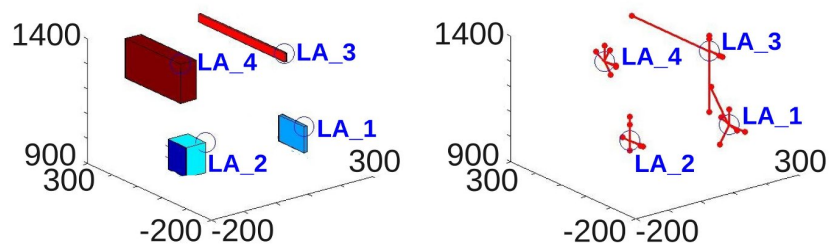

Fig. 9. Simplified Maneuverability Volume $m V$ (left) and Spinning Pencil $s P$ (right) for the arbitrary robot when all joints are working. The Location Area at the top right has low priority, therefore, the disadvantageous $m V$ and $s P$ at LA_3 does hardly carry weight. The corresponding $m V$ has just one long side and the $s P$ is just spanned up in a plane.

Volumes and Spinning Pencils in a simplified, but more illustrative manner. We just show the parallelepiped $K$ as Maneuverability Volume. The remaining parallelepiped $L$ puts an additional emphasis on the angles between the edges, which are already illustrated in $K$. The illustration of the Spinning Pencil is different from Fig. 3: Our aim is an intuitive, compact interpretation of the figures. Hence, we draw the joint axis of each joint. We wish that a large pencil corresponds to a large value of the Spinning Pencil. Therefore, we set the length of the pencil to $\frac{\beta}{e_{i}}$. A disadvantageous large $e_{i}$ results, then, in a short pencil. We use $\beta=50000$.

The change of $m V$ and $s P$ in the case of the failure of one, resp., three joints of the arbitrary robot becomes clear in Fig. 9-11. The robot is loosing its capabilities, especially at the prioritized Location Areas at the bottom. Furthermore, Fig. 11 shows the additional tolerance, which the robot needs to reach the positions at all: The blue circles in Fig. 9 and 10 symbolize the original tolerance. For the purpose of a better illustration, these circles are larger than the original tolerance. The circles in Fig. 11 are proportionally enlarged according to the additional tolerance, which the robot needs to reach the desired position.

Fig. 12 and 13 illustrate results of the PUMA similarly to the previous figures. Since the detail of both figures is the same as for the figures of the arbitrary robot, the larger magnitudes of the simplified $m V$ and $s P$ for the PUMA become visible. Nevertheless, the final results of both 

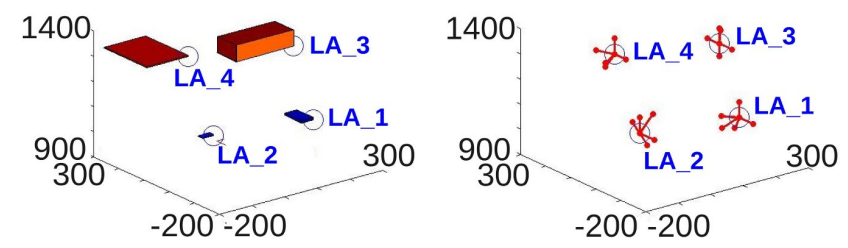

Fig. 10. Simplified $m V$ (left) and $s P($ right) for the arbitrary robot with broken joint 1 . At the important Location Areas at the bottom, the robot can just reach strongly disadvantageous $m V$. Even though the $s P$ is not significantly affected by the joint failure, the bad $m V$ at the bottom reduces the possible maneuverability significantly as the statistics in Fig. 6 show.
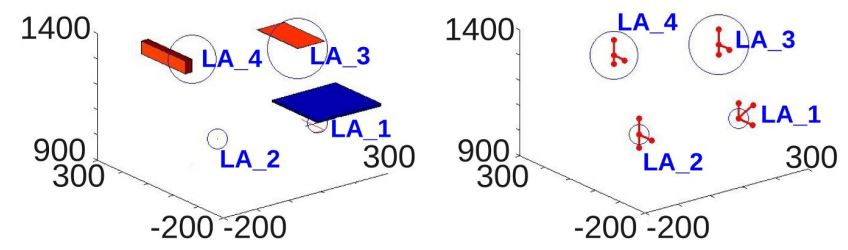

Fig. 11. Simplified $m V$ (left) and $s P($ right $)$ for the arbitrary robot with broken joint 4,5 and 6 . As the larger blue circles at the top Location Areas already indicate, the robot has problems to reach the desired positions. At the same time, the $m V$ are reduced, especially at the bottom left, and the $s P$ are getting smaller.
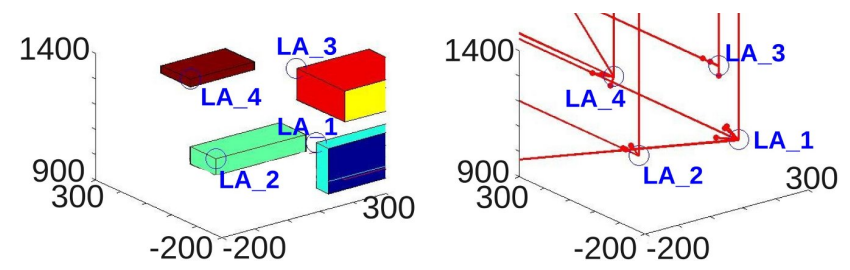

Fig. 12. Simplified $m V$ (left) and $s P$ (right) for the PUMA when all joints are working. The detail of Fig. 9 and this figure is the same to enable an easier comparison, even though the $m V$ and $s P$ do not fit in this figure any more.
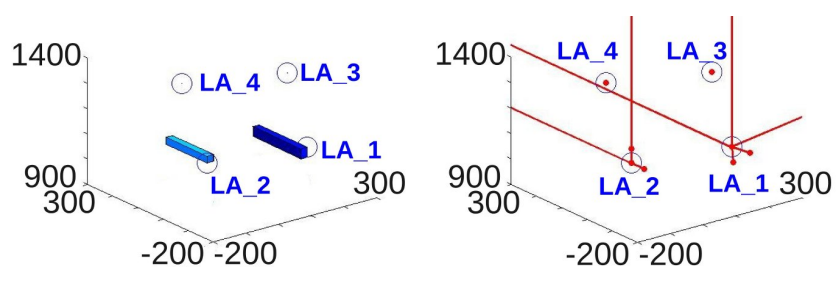

Fig. 13. Simplified $m V$ (left) and $s P$ (right) for the PUMA with broken joint 3 . The robot is not able to reach the less important Location Areas at the top any more, but it can position its end-effector at the prioritized areas at the bottom. The $s P$ is still relatively advantageous there.

entirely working robots in Fig. 6 are nearly the same for both robots. This due to several reasons. First, multiple parts of the PUMA's Spinning Pencils point (nearly) into the same direction. Second, we put an emphasis on $m V$, which is not as large for the PUMA as its Spinning Pencils. Third, the large Maneuverability Volumes at the top are located at a less important Location Areas. Therefore, they influence the final result less significantly.

We repeat the experiments with none, one and two broken joints a second time to check the repeatability of the results. The Stochastic Optimizer in the estimation of the inverse kinematics can lead to slightly different configurations at the desired positions. The repetition of the experiments show, that these differences affect the magnitude of the results of the Maneuverability Analysis just slightly with an average difference of 0.005 , including five outliers with a difference higher than 0.02

\section{CONCLUSION}

We proposed an analysis of the maneuverability of different, arbitrary manipulators under possible joint failures in task-specific contexts. We introduced the Maneuverability Volume and the Spinning Pencil for the abstract representation of the efficiency of the robot's capabilities. The results of our experiments show, (1) whether the specified task areas can be reached under joint failure, (2) the magnitude of the reduction of the original maneuverability under joint failure, (3) the essential joints for the desired task and (4) the illustrative comparison of the capabilities of robots.

In future work, this approach can be extended to manipulators with translational joints and more complex systems like humanoid robots. Moreover, the presented approach can be included into the advantageous design of manipulators with respect to the efficiency in control.

\section{REFERENCES}

[1] S. Petsch and D. Burschka, "Representation of manipulation-relevant object properties and actions for surprise-driven exploration," in IEEE/RSJ International Conference on Intelligent Robots and Systems, San Francisco, California, USA, 2011, pp. 1221-1227.

[2] T. Yoshikawa, "Manipulability of robotic mechanisms," The International Journal of Robotics Research, vol. 4, no. 2, 1985.

[3] J.-O. Kim and P. K. Khosla, "Dexterity measures for design and control of manipulators," in IEEE/RSJ International Workshop on Intelligent Robots and Systems, Osaka, Japan, 1991, pp. 758-763.

[4] C. J. J. Paredis and P. K. Khosla, "Kinematic design of serial link manipulators from task specifications," The International Journal of Robotics Research, vol. 12, no. 274, pp. 273-287, 1993.

[5] H. Asada, "Dynamic analysis and design of robot manipulators using inertia ellipsoids," in IEEE International Conference on Robotics and Automation, 1984, pp. $94-102$.

[6] J. Lee, "A study of the manipulability measures for robot manipulators," in IEEE/RSJ International Conference on Intelligent Robots and Systems, 1997, pp. 1458-1465.

[7] A. Bowling and O. Khatib, "The dynamic capability equations: A new tool for analyzing robotic manipulator performance," IEEE Transactions on Robotics, vol. 21, no. 1, pp. 115 - 123, 2005.

[8] M. Ciocarlie, C. Goldfeder, and P. Allen, "Dimensionality reduction for hand-independent dexterous robot grasping," in IEEE/RSJ International Conference on Intelligent Robots and Systems, San Diego, California, USA, 2007, pp. 3270-3275.

[9] J. Romero, T. Feix, H. Kjellström, and D. Kragic, "Spatio-temporal modeling of grasping actions," in IEEE/RSJ International Conference on Intelligent Robots and Systems, Taipei, Taiwan, 2010, pp. 21032108.

[10] K.-M. Lee and D. K. Shah, "Kinematic analysis of a three-degrees-offreedom in-parallel actuated manipulator," IEEE Journal of Robotics and Automation, vol. 4, no. 3, 1988.

[11] J. Denavit and R. S. Hartenberg, "A kinematic notation for lower-pair mechanisms based on matrices," Journal of Applied Mechanics, pp. 215-221, 1955.

[12] J. J. Craig, Introduction to Robotics - Mechanics and Control. Prentice Hall, 2005.

[13] C. Papazov and D. Burschka, "Stochastic global optimization for robust point set registration," Computer Vision and Image Understanding, vol. 115, December 2011.

[14] O. Ruepp, "Recovery of structure and motion from monocular images under poor lighting and texture conditions," Ph.D. dissertation, Technische Universität München, 2012. 